\title{
Are Dynamic Arterial Spin-Labeling MRA and Time-Resolved Contrast-Enhanced MRA Suited for Confirmation of Obliteration following Gamma Knife Radiosurgery of Brain Arteriovenous Malformations?
}

\author{
(D) A. Rojas-Villabona, (D) F.B. Pizzini, (D)T. Solbach, (D) M. Sokolska, (D) R. Ricciardi, (D) C. Lemonis, (D) E. DeVita, (D) Y. Suzuki, \\ (D) M.J.P. van Osch, (DR.I. Foroni, (D) M. Longhi, (D) Sontemezzi, (D)D. Atkinson, (DN. Kitchen, (D) A. Nicolato, (D) X. Golay, and \\ (D) H.R. Jäger
}

\begin{abstract}
BACKGROUND AND PURPOSE: Intra-arterial DSA has been traditionally used for confirmation of cure following gamma knife radiosurgery for AVMs. Our aim was to evaluate whether 4D arterial spin-labeling MRA and contrast-enhanced time-resolved MRA in combination can be an alternative to DSA for confirmation of AVM obliteration following gamma knife radiosurgery.

MATERIALS AND METHODS: In this prospective study, 30 patients undergoing DSA for confirmation of obliteration following gamma knife radiosurgery for AVMs (criterion standard) also underwent MRA, including arterial spin-labeling MRA and contrastenhanced time-resolved MRA. One dataset was technically unsatisfactory, and the case was excluded. The DSA and MRA datasets of 29 patients were independently and blindly evaluated by 2 observers regarding the presence/absence of residual AVMs.
\end{abstract}

RESULTS: The mean time between gamma knife radiosurgery and follow-up DSA/MRA was 53 months (95\% Cl, 42-64 months; range, 22-168 months). MRA total scanning time was 9 minutes and 17 seconds. Residual AVMs were detected on DSA in 9 subjects (obliteration rate $=69 \%$ ). All residual AVMs were detected on at least 1 MRA sequence. Arterial spin-labeling MRA and contrastenhanced time-resolved MRA showed excellent specificity and positive predictive values individually (100\%). However, their sensitivity and negative predictive values were suboptimal due to 1 false-negative with arterial spin-labeling MRA and 2 with contrastenhanced time-resolved MRA (sensitivity $=88 \%$ and $77 \%$, negative predictive values $=95 \%$ and $90 \%$, respectively). Both sensitivity and negative predictive values increased to $100 \%$ if a composite assessment of both MRA sequences was performed. Diagnostic accuracy (receiver operating characteristic) and agreement $(\kappa)$ are maximized using arterial spin-labeling MRA and contrast-enhanced time-resolved MRA in combination (area under receiver operating characteristic curve $=1, P<.001 ; \kappa=1, P<.001$, respectively).

CONCLUSIONS: Combining arterial spin-labeling MRA with contrast-enhanced time-resolved MRA holds promise as an alternative to DSA for confirmation of obliteration following gamma knife radiosurgery for brain AVMs, having provided $100 \%$ sensitivity and specificity in the study. Their combined use also enables reliable characterization of residual lesions.

ABBREVIATIONS: $\mathrm{ASL}=$ arterial spin-labeling; $\mathrm{CE}=$ time-resolved contrast-enhanced; GKR = gamma knife radiosurgery; $\mathrm{NPV}=$ negative predictive value; PPV = positive predictive value; $\mathrm{ROC}=$ receiver operating characteristic

B rain AVMs are a potential source of neurologic morbidity and mortality due to the life-long risk of intracranial bleeding if left untreated (1.5\%-4.0\% per year). ${ }^{1}$ Gamma knife radiosurgery
(GKR) is a well-established treatment for selected patients with brain AVMs. ${ }^{2}$ It triggers a gradual decrease in blood flow through the AVM nidus a few months after treatment, which progresses so
Received April 10, 2020; accepted after revision October 21, 2021.

From The Gamma Knife Centre at Queen Square (A.R.-V.), The Lysholm Department of Neuroradiology (T.S., H.R.).), and Department of Neurosurgery (N.K.), National Hospital for Neurology and Neurosurgery, London, UK; Department of Neurosurgery (A.R.-V.), Royal Victoria Infirmary, Newcastle upon Tyne, UK; Department of Radiology (F.B.P., R.I.F.), Department of Diagnostic and Public Health, Verona University, Verona, Italy; Department of Medical Physics and Bioengineering (M.S.), Neuroradiological Academic Unit (M.S., X.G., H.R.J.), Department of Brain Repair and Rehabilitation, Institute of Neurology and Centre for Medical Imaging (D.A.), University College London, London, UK; School of Biomedical Engineering and Imaging Sciences (E.D.V.), King's College London, London, UK; Wellcome Centre for Integrative Neuroimaging (Y.S.), FMRIB, Nuffield Department of Clinical Neurosciences, University of Oxford, Oxford, UK; CJ. Gorter Center for High Field MRI (M.J.P.v.O.), Department of Radiology, Leiden University
Medical Center, Leiden, Netherlands; and Department of Neuroscience (M.L., A.N.); and Radiology Unit (S.M.) and Neuroradiology Unit (G.R., C.L.), Department of Diagnostic and Pathology, University Hospital of Verona, Verona, Italy.

This project was funded by the National Brain Appeal (Small Acorns grant), Elekta (UK) Limited, and the Verona Brain Research Foundation. It was also sponsored by the National Institute for Health Research Biomedical Research Centre and Queen Square Radiosurgery Centre.

Please address correspondence to Alvaro Rojas-Villabona, MBBS, MSc, PhD, MRCS, Department of Neurosurgery, Royal Victoria Infirmary, Queen Victoria Rd, Newcastle upon Tyne, NE1 4LP, United Kingdom; e-mail: a.villabona.11@ucl.ac.uk

- Indicates open access to non-subscribers at www.ajnr.org

http://dx.doi.org/10.3174/ajnr.A6990 
that by 2-3 years posttreatment, approximately $75 \%$ of AVMs are completely obliterated. ${ }^{3}$ The risk of intracranial bleeding is thought to persist until complete obliteration of the nidus is achieved, so it is imperative to confirm AVM cure after treatment.

Intra-arterial DSA has been traditionally used for confirmation of obliteration following GKR., ${ }^{4,5}$ Most GKR centers around the world also use regular MRI and MRA for follow-up, but most patients still undergo DSA to confirm a cure a few years after treatment, usually once obliteration is suggested by MRI/MRA. ${ }^{6}$ Although widely used, DSA conveys some risk, which includes neurologic complications (2.63\%), stroke with permanent disability $(0.14 \%)$, death $(0.06 \%)$, and access site hematoma $(4.2 \%){ }^{7}$ DSA also exposes both patients and medical staff to ionizing radiation, and it carries a risk associated with the injection of iodinated contrast agents. It is an unpleasant experience for patients due to pain, invasiveness, and prolonged bed rest after the procedure. ${ }^{8}$ The cost associated with DSA examinations is also significantly larger than that of diagnostic MRI.

The potential to improve follow-up procedures after GKR using less invasive and radiation-free imaging methods has been described elsewhere, and important attempts have been made using MRA as an alternative to DSA. ${ }^{4,5,9,10}$ Multiple studies have concluded that individually, TOF angiography and time-resolved contrast-enhanced MRA (CE-MRA) have good diagnostic accuracy, which supports their addition to the standard follow-up protocols. However, due to their limited sensitivity and specificity, it is still recommended that DSA be performed to confirm AVM obliteration. MR vascular imaging is being continuously developed, with increasing spatial resolution achieved with contrast-based MR angiography and also subsecond temporal resolution with 4D MRA sequences. ${ }^{11,12}$ The introduction of arterial spin-labeling MRA (ASL-MRA), for instance, has also allowed the acquisition of time-resolved cerebral angiography with temporal resolution comparable with that of DSA (100-200 ms), without the administration of contrast agents. ${ }^{13-15}$ This study aimed to evaluate whether dynamic ASL-MRA and CE-MRA in combination can be an alternative to DSA to confirm obliteration of brain AVMs following GKR and to characterize residual AVMs in cases with an incomplete response.

\section{MATERIALS AND METHODS}

\section{Patients}

Adult patients undergoing DSA for assessment of obliteration following GKR for AVMs at Verona University Hospital between November 2014 and December 2016 were prospectively recruited. The decision to perform follow-up DSA was made by the clinical team on the basis of their routine follow-up procedures, which are that DSA is performed once obliteration is demonstrated on regular follow-up MR imaging/MRA or after 4 years if no response or only partial response is seen on MR imaging/MRA.

The study was approved by the Verona and Rovigo Research Ethics Committee (European equivalent to an institutional review board), and all participants gave written consent for MRA to be performed on the same day as DSA. Exclusion criteria included contraindications to MR imaging (ie, pacemakers, metallic implants), patients unable to tolerate MR imaging without sedation/anesthesia, abnormal renal function (estimated glomerular
Table 1: Demographic details and AVM characteristics of the study subjects

\begin{tabular}{lc}
\hline \multicolumn{1}{c}{ Characteristics } \\
\hline Age (mean) (range) (yr) & $37(18-69)$ \\
Female (\%) & $66 \%$ \\
AVM location (No.) (\%) & $9(30 \%)$ \\
Temporal & $6(20 \%)$ \\
Frontal & $6(20 \%)$ \\
Parietal & $3(10 \%)$ \\
Occipital & $4(13 \%)$ \\
Basal ganglia/brain stem & $2(7 \%)$ \\
Posterior fossa & \\
Lateralization (\%) & $30 \%$ \\
Right & $70 \%$ \\
Left & $7.01(0.07-50.54)$ \\
AVM volume (mean) (range) (mL) & \\
GKR (mean) (range) & $17(11-22)$ \\
Dose (Gy) & $50 \%$ \\
Percentage isodose & $53(22-168)$ \\
Mean time post-GKR (range) (mo) & \\
\hline
\end{tabular}

filtration rate of $<30 \mathrm{~mL} / \mathrm{min}$ ), inability to consent, pregnancy, and history of an allergic reaction to gadolinium.

Thirty consecutive patients were enrolled, and Table 1 shows their demographic details and AVM characteristics. The mean age was 37 years (range, 18-69 years), and 66\% of participants were female. Twenty-two patients (73\%) presented with a ruptured AVM on diagnosis. The mean AVM volume at the time of GKR was 7.01 [SD, 9.8] $\mathrm{mL}$ (range, 0.07-50.54 $\mathrm{mL}$ ), and the AVMs were well-distributed across the head anatomy. Ten patients (33.3\%) had previously undergone glue embolization ( $N$-butyl cyanoacrylate), and 3 (10\%), microsurgical resection before GKR. One of them had undergone GKR twice for the same AVM.

In 1 case, the contrast agent was not detected by CE-MRA due to mis-timing of the gadolinium injection with respect to the dynamic acquisition. This dataset was considered technically unsatisfactory, and the case was excluded. Final statistical analysis included 29 patients.

\section{MRA}

MRA included 4D-ASL-MRA and CE-MRA. These were acquired using an 8-channel head coil on an Achieva 3T MR imaging system (Philips Healthcare) and the scanning parameters shown in Table 2.

ASL-MRA labeling was performed with signal targeting with alternating radiofrequency and a labeling slab of $300 \mathrm{~mm}$ positioned $20 \mathrm{~mm}$ below the imaging plane. ${ }^{16}$ An EPI readout ${ }^{17}$ enabled acquisition of 8 dynamic phases with a temporal resolution of $200 \mathrm{~ms}$. CE-MRA included 24 dynamic sagittal acquisitions using a 3D, T1weighted, fast-field echo sequence. Intravenous injection of $0.1 \mathrm{~mL} /$ $\mathrm{kg}$ of gadobutrol, $1.0 \mathrm{mmol}$ (Gadovist; Bayer Schering Pharma), was administered with an automated injector at $3.5 \mathrm{~mL} / \mathrm{s}$ followed by $20-\mathrm{mL}$ normal saline flush at the same rate. A reference scan was acquired before contrast injection for subtraction of stationary tissue, and dynamic sampling was started at the same time as the injection. The dynamic acquisition used contrast-enhanced robusttiming angiography and the keyhole method, with $20 \%$ of the $k$-space collected per frame, achieving a temporal resolution of $608 \mathrm{~ms} /$ phase. MIPs of the individual MRA sequences were 
Table 2: Scanning parameters of ASL-MRA and CE-MRA

\begin{tabular}{lcc}
\hline \multicolumn{1}{c}{ Parameter } & ASL-MRA & CE-MRA \\
\hline MRA type & Dynamic (4D) & Dynamic (4D) \\
Scan duration (min:sec) & $5: 58$ & $3: 19$ \\
Contrast & & \\
Acquisition & T1-TFEPI & T1-FFE \\
TR (ms) & 12 & 3 \\
TE (ms) & 5 & 1 \\
Flip angle & $10^{\circ}$ & $25^{\circ}$ \\
Resolution & & \\
FOV (RL $\times$ AP $\times$ CC mm) & $210 \times 210 \times 90$ & $150 \times 210 \times 210$ \\
Acquisition & Transversal & Sagittal \\
Slabs & 1 & 1 \\
Acquisition matrix & $172 \times 172 \times 70$ & $50 \times 248 \times 248$ \\
Acquired voxel size (mm) & $1.22 \times 1.26 \times 1.3$ & $3 \times 0.85 \times 0.85$ \\
Reconstruction matrix & $256 \times 256$ & $288 \times 288$ \\
reconstructed voxel size (mm) & $0.82 \times 0.82 \times 0.65$ & $1.5 \times 0.73 \times 0.73$ \\
No. of slices & 140 & 100 \\
SENSE factor & $2.5 / 1$ & $4 / 2$ \\
Dynamic acquisition & & \\
Dynamic imaging mode & TFEPI & CENTRA keyhole \\
No. of dynamic phases & 8 & 24 \\
Phase interval (temporal resolution) (ms) & 200 & 608 \\
Label delay (ms) & 200 & - \\
Contrast & - & Gadovist $0.1 \mathrm{~mL} / \mathrm{Kg} ; \mathrm{IV} \mathrm{pump}$ \\
& & injection: $3.5 \mathrm{~mL} / \mathrm{s}$ \\
\hline No:-icates non
\end{tabular}

Note:-indicates non-applicable; TFEPI, Turbo-field echo-planar imaging; FFE, fast-field echo; CENTRA, enhanced robust-timing angiography; RL, right-left; AP, anterior-posterior; CC, caudo-cranial; SENSE, sensitivity encoding.
$<3 \mathrm{~cm}, 3-6 \mathrm{~cm}$, and $>6 \mathrm{~cm}$; venous drainage: deep versus superficial only; adjacent brain: eloquent versus noneloquent) was used to grade residual lesions. Further characterization of residual AVMs included the identification of feeding arteries and draining veins.

DSA was regarded as the criterion standard. The sensitivity, specificity, positive predictive value (PPV), and negative predictive value (NPV) of ASL-MRA, CEMRA and the combination of both for detection of residual AVMs were calculated. Their diagnostic performance (accuracy) to discriminate between patients with residual AVMs and those with complete obliteration was evaluated using receiver operating characteristic (ROC) curve analysis. An area under the ROC curve of 1 denoted complete agreement, while 0.5 was no agreement other than what would be expected by chance. The weighed Cohen $\kappa$ coefficient was calculated to measure agreement between DSA and MRA ( $\kappa \leq 0.20=$ poor; $\geq 0.91=$ excellent). ${ }^{18}$ Statistical significance was defined as $P<.05$, and analyses were performed using the Statistical Package for the Social Sciences (Version 23; IBM).

\section{RESULTS}

The mean time between GKR and follow-up DSA/MRA was 53 months (95\% CI, 42-64 months; range, 22-168 months). ASLMRA and CE-MRA scanning time was 5 minutes and 58 seconds and 3 minutes and 19 seconds, respectively. At follow-up imaging, no patients had received further treatment for residual AVMs nor had they experienced any intracranial bleeds post-GKR.

\section{Confirmation of AVM Obliteration}

A residual AVM was detected on DSA in 9 subjects, and complete radiographic obliteration was confirmed in 20 patients by both observers using DSA, resulting in an obliteration rate of $69 \%$, which was in complete agreement with the clinical reports produced by the interventional radiologist who had performed the DSA.

The residual AVM was detected by observers on ASL-MRA in 8 subjects and on CE-MRA in 7 of 9 patients who had been shown to have a residual on DSA. Table 3 shows how all residual AVMs were detected on at least $1 \mathrm{MRA}$ sequence. The case in which ASLMRA failed to show the residual AVM (case 30) is presented in Fig 1. In 2 cases, the residual lesion was not observed on CE-MRA (Fig 2) due to the very small size of the residual lesion ( $6 \mathrm{~mm}$, case 13$)$ and the diffuse nature of the also very small residual nidus in case 9. No abnormalities were observed on MRA in those patients with complete radiographic obliteration on DSA. 
Table 3: Identification of residual AVM with DSA, ASL-MRA, and CE-MRA in cases with a residual lesion

\begin{tabular}{lccc}
\hline & \multicolumn{3}{c}{ Residual AVM } \\
\cline { 2 - 4 } Case & DSA & ASL-MRA & CE-MRA \\
\hline 3 & Yes & Yes & Yes \\
6 & Yes & Yes & Yes \\
9 & Yes & Yes & No $^{a}$ \\
10 & Yes & Yes & Yes \\
11 & Yes & Yes & Yes \\
13 & Yes & Yes & No $^{\text {a }}$ \\
19 & Yes & Yes & Yes \\
23 & Yes & Yes & Yes \\
30 & Yes & No $^{\text {a }}$ & Yes \\
\hline
\end{tabular}
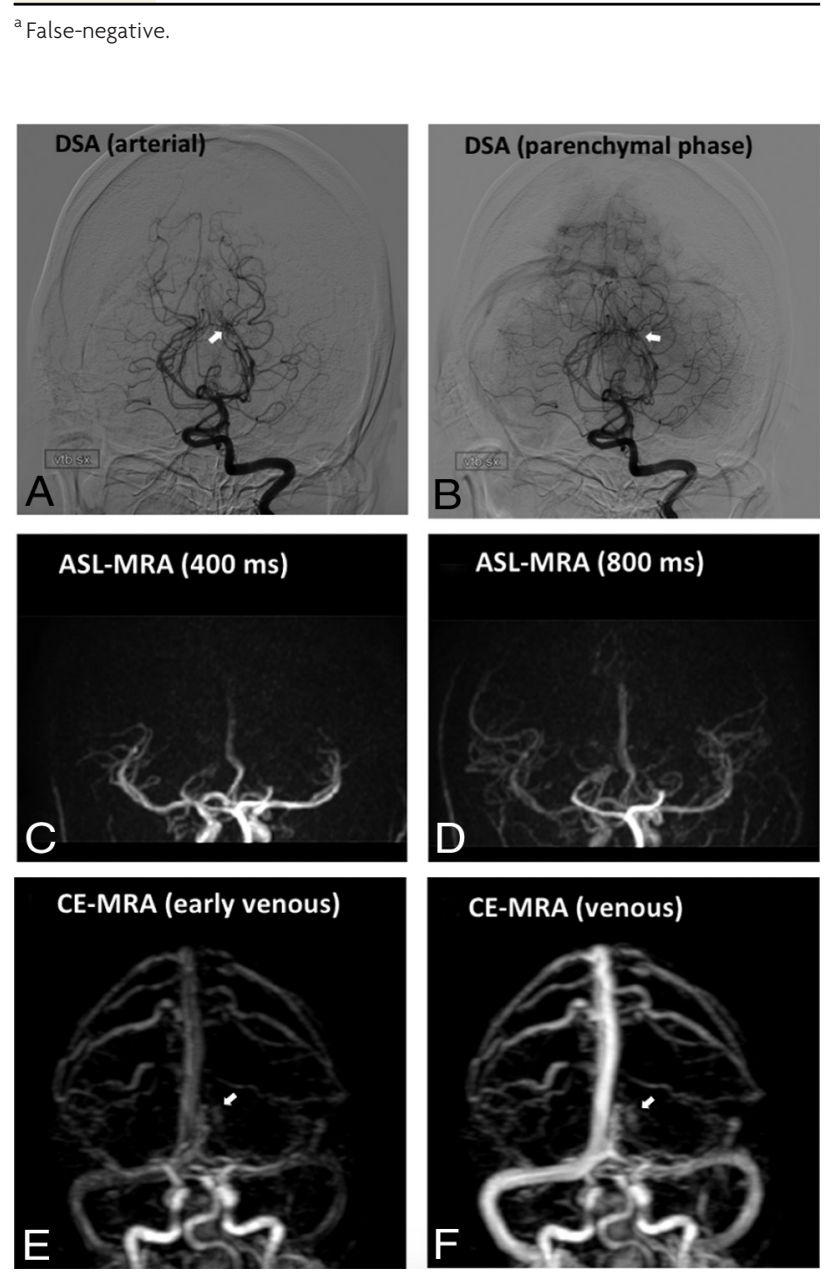

FIG 1. Post-GKR DSA and MRA of a study case in which the residual AVM was not visualized on ASL-MRA (case 30). Post-GKR DSA ( $A$ and $B$ ) shows a residual AVM nidus (white arrow) fed by the left posterior cerebral artery. CE-MRA $(E-F)$ shows slow filling of the AVM nidus (white arrow) lateral to the deep venous system. No residual AVM is noted on ASL-MRA ( $C$ and $D)$.

The sensitivity, specificity, PPV, and NPV of ASL-MRA and CE-MRA for detection of residual AVMs are presented in Table 4. ASL-MRA and CE-MRA showed excellent specificity and PPV individually due to the absence of false-positives. Their sensitivity and NPV, however, were suboptimal due to the finding of 1 falsenegative with ASL-MRA and 2 with CE-MRA (sensitivity $=88 \%$ and $77 \%$; NPV $=95 \%$ and $90 \%$, respectively). Both sensitivity and NPV increased to $100 \%$ if a composite assessment of both
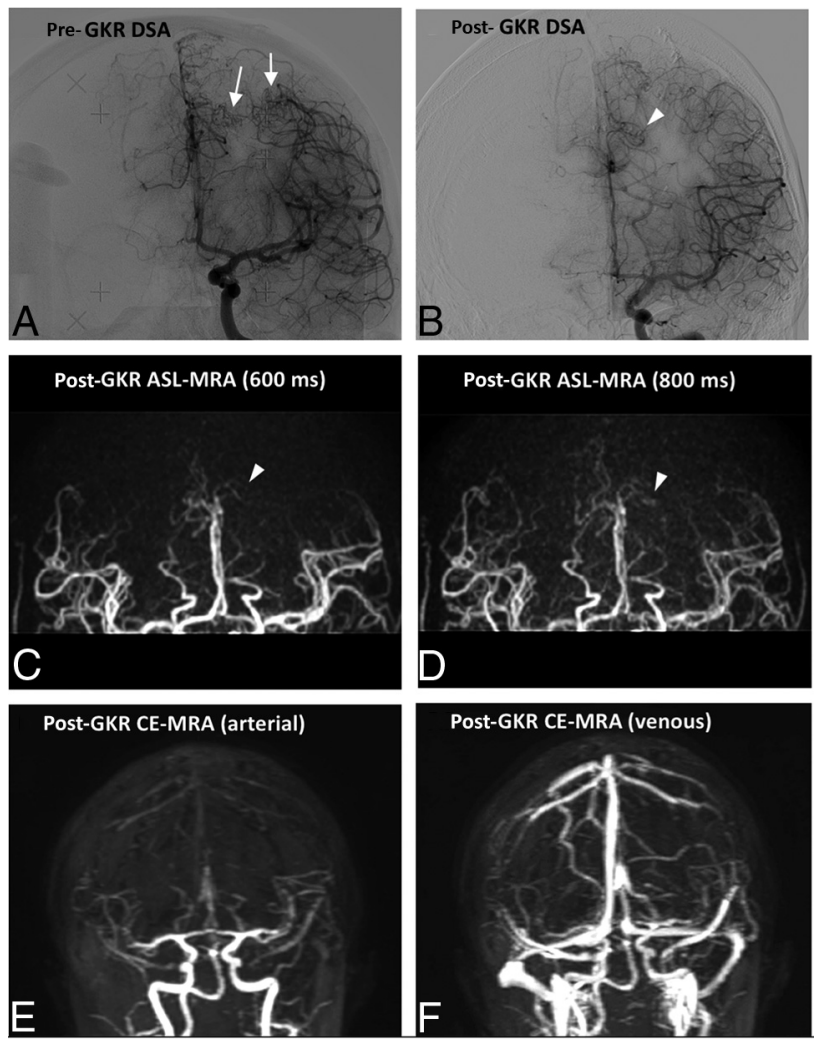

FIG 2. Representative case of a residual AVM not visualized on CEMRA (case 13). The pre-GKR DSA (A) shows 2 AVM nidi (white arrows) at the time of GKR in a patient with previous partial surgical excision of a ruptured AVM. The most lateral nidus is not identified in post-GKR imaging; however, a residual of the most medial nidus (arrowheads) is identified on post-GKR DSA $(B)$ and ASL-MRA $(C$ and $D)$. The small size of the lesion renders it not identifiable on the CE-MRA $(E-F)$.

MRA sequences was performed. Diagnostic accuracy (ROC) and agreement $(\kappa)$ are also maximized using ASL-MRA and CEMRA in combination (area under the ROC curve $=1, P<.001$; $\kappa=1, P<.001$, respectively).

\section{Characterization of Residual AVMs in Cases of Incomplete Response}

Table 5 shows the interpretation of DSA and MRA regarding Spetzler-Martin grading of residual AVMs by both observers. There was complete agreement on size and drainage scores between MRA and DSA for both observers in all 9 residual lesions $(\kappa=1 ; P<.003)$. Different eloquence scores were noted on DSA and MRA in 1 case by the first observer and in 3 cases by the second observer. These were the result of limited anatomic localization on 2D DSA images compared with MRA. Figure 3 shows a representative case of a residual AVM that was fully and accurately characterized in terms of Spetzler-Martin grading using ASL- and CE-MRA compared with DSA.

Observer 1 identified a total of 14 feeding arteries and 13 draining veins on both DSA and MRA. Observer 2 identified 17 feeding arteries and 15 draining veins on DSA and 15 and 12 on MRA, respectively (Table 3 ). The same blood vessel was identified by both observers as the main feeding artery 
Table 4: Sensitivity, specificity, PPV, and NPV of ASL-MRA, CE-MRA, and the combination of the $\mathbf{2}$ for detection of residual AVMs

\begin{tabular}{lccc}
\hline & ASL-MRA & CE-MRA & Both \\
\hline Sensitivity & $88 \%$ & $77 \%$ & $100 \%$ \\
Specificity & $100 \%$ & $100 \%$ & $100 \%$ \\
PPV & $100 \%$ & $100 \%$ & $100 \%$ \\
NPV & $95 \%$ & $90 \%$ & $100 \%$ \\
Diagnostic accuracy (ROC) & $0.94(P<.001)^{\mathrm{a}}$ & $0.89(P=.002)^{\mathrm{a}}$ & $1(P<.001)^{\mathrm{a}}$ \\
Agreement $(\kappa)$ & $0.92(P<.001)$ & $0.82(P<.001)$ & $1(P<.001)$ \\
\hline
\end{tabular}

${ }^{a}$ Null hypothesis: true area $=0.5$.

Table 5: Characterization of residual AVMs using ASL-MRA and CE-MRA compared with DSA

\begin{tabular}{|c|c|c|c|c|}
\hline & \multicolumn{2}{|c|}{ Observer 1} & \multicolumn{2}{|c|}{ Observer 2} \\
\hline & DSA & MRA & DSA & MRA \\
\hline \multicolumn{5}{|l|}{ SMS } \\
\hline \multicolumn{5}{|l|}{ Size } \\
\hline$<3 \mathrm{~cm}$ & 7 & 7 & 7 & 7 \\
\hline $3-6 \mathrm{~cm}$ & 2 & 2 & 2 & 2 \\
\hline$>6 \mathrm{~cm}$ & - & - & - & - \\
\hline \multicolumn{5}{|l|}{ Drainage } \\
\hline Superficial only & 5 & 5 & 5 & 5 \\
\hline Deep & 4 & 4 & 4 & 4 \\
\hline \multicolumn{5}{|l|}{ Eloquence } \\
\hline Noneloquent & 5 & 4 & 3 & 4 \\
\hline Eloquent & 4 & 5 & 6 & 5 \\
\hline Feeding arteries & 14 & 14 & 17 & 15 \\
\hline Draining veins & 13 & 13 & 15 & 12 \\
\hline
\end{tabular}

Note:-indicates non-applicable; SMS, Spetzler-Martin Score.

on DSA and MRA for 8 of 9 residual AVMs. In 1 case, observer 1 named the superior cerebellar artery as the only feeder on DSA, and the posterior cerebral artery, on MRA, while observer 2 named the same feeding vessels but in the opposite set of investigations, ie, superior cerebellar artery on MRA and posterior cerebral artery on DSA.

\section{DISCUSSION}

This work presents the use of 2 dynamic MRA techniques based on ASL and gadolinium bolus tracking for confirmation of obliteration of brain AVMs following GKR. The ASL-MRA, CEMRA, and DSA sequences of 29 patients who underwent followup imaging to confirm obliteration of brain AVMs following GKR were blindly and independently assessed by 2 observers. ASL-MRA and CE-MRA individually have limited sensitivity and NPV for detection of residual AVMs after radiosurgery. This is of clinical importance because false-negative investigations could result in a persistent risk of hemorrhage from AVMs in patients thought to have been cured. The combined use of ASL-MRA and CE-MRA, which provide both temporal and spatial information, appears to be a reliable method to confirm/rule out the presence of residual AVMs after radiosurgery. The composite use of dynamic ASL-MRA and CE-MRA in our study showed maximal sensitivity and specificity compared with DSA; this outcome makes this the first study demonstrating satisfactory diagnostic accuracy of MRA for confirmation of obliteration following GKR of brain AVMs. The results of this study support the use of ASL-
MRA and CE-MRA as a first-line technique for confirmation of obliteration following GKR, which is of high clinical relevance because this would not only avoid unnecessary DSA examinations for patients with cured AVMs (69\% in our study group) but also help rationalize its use in patients with residual AVMs who may need more DSAs for further treatment.

The major accomplishment of using two 4D-MRA sequences is the combination of high temporal and spatial resolution, which is necessary to appreciate the dynamic characteristics of AVMs. ${ }^{19}$ AVMs exhibit a high degree of heterogeneity in terms of angioarchitecture and flow dynamics, and even within the same lesion, there may be dramatic and unpredictable differences in terms of vessel configuration and blood flow. This accomplishment is most likely the reason for the disappointing results of previous studies that attempted to detect and fully characterize residual AVMs using either TOF or CEMRA. $^{4,5,9,10}$ If used independently, those MRA sequences are unable to capture all the complexity and heterogeneity of shapes, sizes, and velocities seen on AVMs. However, in combination, the chance of false-negatives is reduced and their combined ability to detect/rule out residual AVMs after GKR is increased. This feature is demonstrated in our study by the visualization of all residual AVMs in at least 1 MRA sequence and the optimal characterization of residual AVMs using structural and dynamic information obtained from both MRA modalities. Such use of multiple sequences for diagnostic purposes is commonplace in radiology, and it is well-accepted that information from multiple sources (ie, T1WI, T2WI, and contrast imaging) may be required to achieve a full diagnosis.

The main limitation of our study is that a separate review of each MRA sequence, to assess their ability to individually confirm AVM obliteration, was not part of the design. This was because multiple studies had previously concluded that individually, all previously used MRA sequences have suboptimal diagnostic accuracy compared with DSA. ${ }^{4,5,9,10}$ Also, the review of both MRA sequences separately followed by their combined re-assessment would have resulted in increasing and unpredictable reporting bias. Instead, as part of the combined assessment of both MRA sequences, the observers indicated which of the 2 showed the residual AVM, and this information provided some insight into the diagnostic accuracy of each individual sequence. However, we cannot assume that the outcome would have been the same if each MRA technique were assessed independently because the combined assessment of the 2 is highly likely to have resulted in a degree of crossed reinforcement or reassurance of findings between the individual sequences.

In our study, CE-MRA appeared to have lower diagnostic accuracy by failing to show 2 residual AVMs that were clearly visualized on ASL-MRA and DSA (Fig 2). CE-MRA is prone to motion artifacts, and it relies on single-pass bolus tracking, which contributed to a technically unsatisfactory MRA dataset that was excluded from the analysis. Nonetheless, CE-MRA provided 

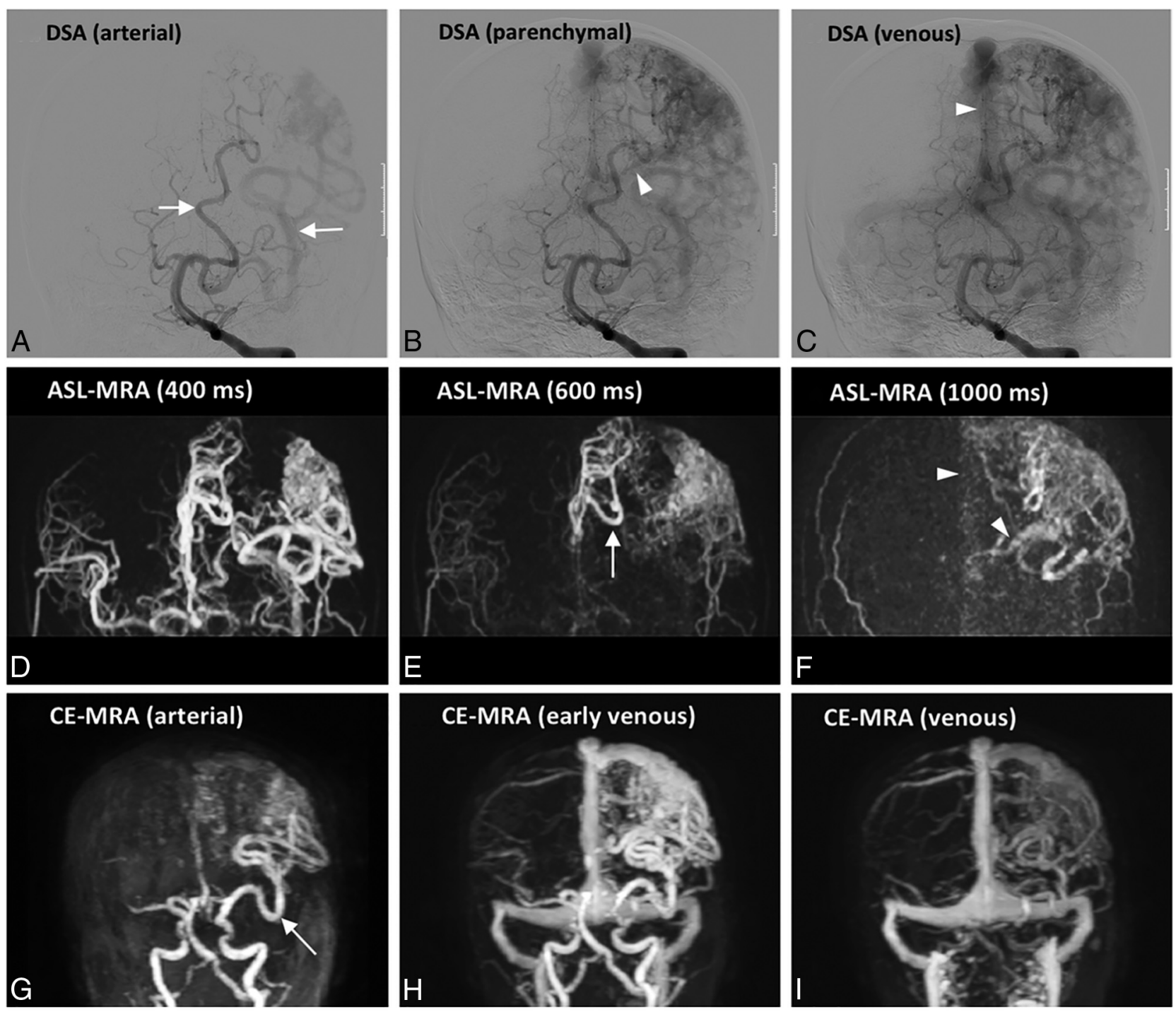

FIG 3. Characterization of a residual AVM (case 19) using ASL $(D-F)$ and CE-MRA $(G-I)$ compared with DSA $(A-C)$. The feeding arteries of this AVM, which are branches of the anterior and middle cerebral arteries (white arrows), are clearly depicted on ASL-MRA and CE-MRA . The draining veins (arrowheads) seen on DSA can also be identified on ASL and CE-MRA, and they involve both the superficial venous system via the superior sagittal sinus and the deep system via the left internal cerebral vein.

valuable dynamic information for the characterization of residual lesions, in terms of feeding arteries and draining veins, and it can also depict arteriovenous shunting, which was essential in the evaluation of AVM obliteration. CE-MRA was very important in a case in which the residual AVM was not visualized on ASL-MRA (Fig 1), and this case demonstrates how the 2 sequences complement each other, increasing the chances of AVM detection and enabling the characterization of residual AVMs.

Regarding ASL-MRA, to date, no previous studies exist on its use for confirmation of AVM obliteration after radiosurgery. A number of studies have used it, together with ASL perfusion, for assessment of AVMs with promising results, but their value compared with DSA is also still to be fully established. ${ }^{20-22}$ ASL is an appealing technique for confirmation of obliteration because venous signal in AVM draining veins can be considered a strong indication of arteriovenous shunting, which is an unequivocal defining element of an AVM. During an ASL acquisition, the arterial blood water is labeled with a radiofrequency pulse proximal to the brain, and it is used as an endogenous contrast agent. ${ }^{17,23}$ Under normal physiologic conditions, the time it would take for the labeled water to reach the veins (either via intravascular transport through the microvascular bed or via extravasation into the extravascular compartment and subsequently being picked up in the venous system) is longer than the time between the start of labeling and the readout. Thus, there is normally little detectable signal within the intracranial veins on ASL perfusion images ${ }^{24}$ or on ASL angiography. Furthermore, decay of the tagged spins in ASL angiography is accelerated by the train of readout excitation pulses, resulting in additional signal reduction more distal into the vascular tree. In AVMs, however, the labeled spins are rapidly shunted directly into the venous circulation, giving rise to high signal intensity in AVM draining veins. ${ }^{25}$ In our study, ASLMRA proved critical to the assessment of the response to GKR in 
2 cases in which CE-MRA failed to demonstrate the residual lesion (Fig 2). It also provided important information on nidus size and the number of feeding arteries. However, as in other individual MRA sequences, ASL-MRA is, by itself, unable to capture all the possible scenarios of size, flow, and velocity to reliably detect residual AVMs, evidenced by the occurrence of a false-positive in which ASL-MRA failed to show the residual nidus (Fig 1).

In our study, the observers were aided by a very high index of suspicion because they were aware of the location of AVMs at treatment and, therefore, knew where to look for residual lesions. It could be argued that results would have been different if the observers had no indication of the potential AVM location; however, the study was pragmatically designed to replicate the clinical scenario in which DSA examinations are reviewed specifically to confirm obliteration. We performed DSA once obliteration was demonstrated on regular follow-up MR imaging/MRA or after 4 years if no response or only partial response was seen on MR imaging/ MRA. This procedure could have introduced a degree of selection bias. Also, we had a relatively small sample size, which is a limitation of the study. The assessment of a larger number of patients is needed to strengthen the power of the results.

Optimization of MRA sequences for further implementation should include individualized time resolution of ASL-MRA, which can be used to better characterize the flow dynamics of residual AVMs. There is also a great potential for improvement of MRA by postprocessing the individual datasets and, through their combination, generating a single product that incorporates the information provided by both sequences. This improvement is still to be accomplished and developers should aim for more sophisticated $4 \mathrm{D}$ visualization maps, which enhance the visual experience and facilitate interpretation.

\section{CONCLUSIONS}

Combining ASL-MRA with CE-MRA holds promise as an alternative to DSA for confirmation of obliteration following GKR for brain AVMs, having provided $100 \%$ sensitivity and specificity in the study. In addition, their combined use enables reliable characterization of residual lesions.

\section{ACKNOWLEDGMENTS}

We thank Ing Marcello Cadioli, MR Clinical Scientist, for his support on the implementation of CE-MRA at the University Hospital of Verona.

Disclosures: Alvaro Rojas-Villabona-RELATED: Grant: National Brain Appeal, Comments: Small Acorns grant awarded to Alvaro Rojas-Villabona*; Support for Travel to Meetings for the Study or Other Purposes: Queen Square Radiosurgery Center. Matthias J.P. van Osch-UNRELATED: Grants/Grants Pending: Philips Healthcare*; Payment for Lectures Including Service on Speakers Bureaus: Philips Healthcare. Xavier Golay-UNRELATED: Employment: University College London. Hans Rolf Jäger-UNRELATED: Royalties: payment by Springer of $£ 1000$, Comments: This is an honorarium for coediting the 3-volume textbook Clinical Neuroradiology, published by Springer in 2019. Giuseppe Ricciardi-UNRELATED: Employment: University of Verona; Travel/ Accommodations/Meeting Expenses Unrelated to Activities Listed: University of Verona. Christos Lemonis-RELATED: Grant: Verona Brain Research. *Money paid to the institution.

\section{REFERENCES}

1. da Costa L, Wallace MC, Ter Brugge KG, et al. The natural history and predictive features of hemorrhage from brain arteriovenous malformations. Stroke 2009;40:100-05 CrossRef Medline

2. van Beijnum J, van der Worp HB, Buis DR, et al. Treatment of brain arteriovenous malformations: a systematic review and meta-analysis: Meta-Analysis Research Support, Non-U.S. Gov't Review. JAMA 2011;306:2011-19 CrossRef Medline

3. Koltz MT, Polifka AJ, Saltos A, et al. Long-term outcome of gamma knife stereotactic radiosurgery for arteriovenous malformations graded by the Spetzler-Martin classification. J Neurosurg Sci 2013;118:74-83 CrossRef Medline

4. Lee CC, Reardon MA, Ball BZ, et al. The predictive value of magnetic resonance imaging in evaluating intracranial arteriovenous malformation obliteration after stereotactic radiosurgery. J Neurosurg 2015;123:136-44 CrossRef Medline

5. Soize S, Bouquigny F, Kadziolka K, et al. Value of 4D MR angiography at $3 \mathrm{~T}$ compared with DSA for the follow-up of treated brain arteriovenous malformation. AJNR Am J Neuroradiol 2014;35:1903-09 CrossRef Medline

6. Starke RM, Kano H, Ding D, et al. Stereotactic radiosurgery for cerebral arteriovenous malformations: evaluation of long-term outcomes in a multicenter cohort. J Neurosurg Sci 2017;126:36-44 CrossRef Medline

7. Kaufmann TJ, Huston J 3rd, Mandrekar JN, et al. Complications of diagnostic cerebral angiography: evaluation of 19,826 consecutive patients. Radiology 2007;243:812-19 CrossRef Medline

8. Fifi JT, Meyers PM, Lavine SD, et al. Complications of modern diagnostic cerebral angiography in an academic medical center. $J$ Vasc Interv Radiol 2009;20:442-47 CrossRef Medline

9. Buis DR, Bot JC, Barkhof F, et al. The predictive value of $3 \mathrm{D}$ timeof-flight MR angiography in assessment of brain arteriovenous malformation obliteration after radiosurgery. AJNR Am J Neuroradiol 2012;33:232-38 CrossRef Medline

10. Lim HK, Choi CG, Kim SM, et al. Detection of residual brain arteriovenous malformations after radiosurgery: diagnostic accuracy of contrast-enhanced four-dimensional MR angiography at 3.0 T. $\mathrm{Br}$ J Radiol 2012;85:1064-69 CrossRef Medline

11. Chang $\mathrm{W}, \mathrm{Wu} \mathrm{Y}$, Johnson $\mathrm{K}$, et al. Fast contrast-enhanced 4D MRA and 4D flow MRI using constrained reconstruction (HYPRFlow): potential applications for brain arteriovenous malformations. AJNR Am J Neuroradiol 2015;36:1049-55 CrossRef Medline

12. Hadizadeh DR, Gieseke J, Beck G, et al. View-sharing in keyhole imaging: Partially compressed central k-space acquisition in timeresolved MRA at 3.0 T. Eur J Radiol 2011;80:400-06 CrossRef Medline

13. Wu H, Block WF, Turski PA, et al. Noncontrast dynamic 3D intracranial MR angiography using pseudo-continuous arterial spin labeling (PCASL) and accelerated 3D radial acquisition. J Magn Reson Imaging 2014;39:1320-26 CrossRef Medline

14. Lindner T, Jensen-Kondering $U$, van Osch MJ, et al. 3D timeresolved vessel-selective angiography based on pseudo-continuous arterial spin-labeling. Magn Reson Imaging 2015;33:840-06 CrossRef Medline

15. Suzuki $Y$, Teeuwisse M, Schmid S. Improving 4D pCASL angiography by combining Hadamard time-encoding with Look-Locker readout. In: Proceedings of the 22th Annual Meeting of the International Society of Magnetic Resonance in Medicine, Milan, Italy, 2014

16. Nakamura M, Yoneyama M, Tabuchi T, et al. Non-contrast timeresolved magnetic resonance angiography combining high resolution multiple phase echo planar imaging based signal targeting and alternating radiofrequency contrast inherent inflow enhanced multi phase angiography combining spatial resolution echo planar imaging based signal targeting and alternating radiofrequency in intracranial arteries [in Japanese]. Nihon Hoshasen Gijutsu Gakkai Zasshi 2012;68:1525-32 CrossRef Medline

17. Suzuki Y, Fujima N, Ogino T, et al. Acceleration of ASL-based timeresolved MR angiography by acquisition of control and labeled 
images in the same shot (ACTRESS). Magn Reson Med 2018;79:22433 CrossRef Medline

18. Machet A, Portefaix C, Kadziolka K, et al. Brain arteriovenous malformation diagnosis: value of time-resolved contrast-enhanced MR angiography at 3.0T compared to DSA. Neuroradiology 2012;54:1099-108 CrossRef Medline

19. Khandanpour N, Griffiths P, Warren D, et al. Prospective comparison of late 3T MRI with conventional angiography in evaluating the patency of cerebral arteriovenous malformations treated with stereotactic radiosurgery: comparative study controlled clinical trial. Neuroradiology 2013;55:683-87 CrossRef Medline

20. Iryo Y, Hirai T, Nakamura M, et al. Evaluation of intracranial arteriovenous malformations with four-dimensional arterial-spin labeling-based 3-T magnetic resonance angiography. J Comput Assist Tomogr 2016;40:290-96 CrossRef Medline

21. Fujima N, Osanai T, Shimizu Y, et al. Utility of noncontrastenhanced time-resolved four-dimensional MR angiography with a vessel-selective technique for intracranial arteriovenous malformations. J Magn Reson Imaging 2016;44:834-45 CrossRef Medline
22. Yu S, Yan L, Yao Y, et al. Noncontrast dynamic MRA in intracranial arteriovenous malformation (AVM), comparison with time of flight (TOF) and digital subtraction angiography (DSA): Comparative Study Research Support, N.I.H., Extramural Research Support, Non-U.S. Magn Reson Imaging 2012;30:86977 CrossRef Medline

23. Petersen ET, Zimine I, Ho Y-CL, et al. Non-invasive measurement of perfusion: a critical review of arterial spin labelling techniques. other. $R$ J Radiol 2006;79:688-701 CrossRef Medline

24. Kukuk GM, Hadizadeh DR, Bostrom A, et al. Cerebral arteriovenous malformations at 3.0 $\mathrm{T}$ : intraindividual comparative study of 4DMRA in combination with selective arterial spin labeling and digital subtraction angiography: comparative study. Invest Radiol 2010;45:126-32 CrossRef Medline

25. Jang J, Schmitt P, Kim BY, et al. Non-contrast-enhanced 4D MR angiography with STAR spin labeling and variable flip angle sampling: a feasibility study for the assessment of dural arteriovenous fistula. Neuroradiology 2014;56:305-14 CrossRef Medline 